\title{
A Hybrid Approach for Assessments of Equivalent Emission Sources and Electromagnetic Environments
}

\author{
D. V. F. Cardia ${ }^{1}$, C. A. F. Sartori ${ }^{1,2}$, R. P. B. Muylaert ${ }^{1}$ \\ ${ }^{l}$ PEA/EPUSP - Escola Politécnica da Universidade de São Paulo, Departamento de Engenharia Elétrica \\ ${ }^{2}$ IPEN/CNEN-SP - Instituto de Pesquisas Energéticas e Nucleares [São Paulo] \\ daniel.cardia@usp.br, sartori@pea.usp.br,rafael@mbm.eng.br
}

\begin{abstract}
This paper presents a method for equivalent emission source determination by application of LLAs (Large Loop Antennas). A review of different types of LLAs is carried out and their characteristics regarding emission source evaluation are emphasized. A hybrid technic is applied for obtaining the electric fields based on previous measurement of magnetic fields combined with an analytical and numerical approach. This methodology is proposed as an alternative to face the constraints concerning to the near electric fields measurements and calculations.
\end{abstract}

Index Terms-Theoretical and Numerical Methods, Field Measurements, Large Loop Antennas, Equivalent Emission Sources, Near Field Calculation.

\section{INTRODUCTION}

The technological advancements bring to the society an immense quantity of benefits, as: modern communication systems, comfort, transportation, etc. However, additionally, it leads to an increasingly congested electromagnetic environment. Historically, the effects of exposition to electromagnetic fields are being intensively discussed, in order to determine the acceptable limits of the fields emitted by a certain source or supported by an electric or electronic device (immunity). For certain areas of engineering, as automotive, the electromagnetic emission limits are referenced by several standards that regulate the well known electromagnetic compatibility (EMC) tests [1], [2].

The electromagnetic fields measurement in anechoic or shielded chambers is the most usual technic applied for radiated EMC evaluation. Nevertheless, this technic requires a large quantity of equipment and infrastructure. Additionally, this technic requires a real (physical) model of the device that will be tested, which is usually available only in intermediate phases of design. It means that, in case of a test reproval and consequent re-design, a large amount of funding is necessary, because of the spending with new prototypes and re-tests.

In this mean, hybrid methods using measurements, analytical and numerical calculation can be suitable for supporting EMC evaluations in earlier phases of a device design and allowing a larger number of models to be tested and optimized.

During the last years, Large Loop Antennas are being intensively studied as a method of measurement of electric and electronic devices. Alternatively, this type of antenna allows the determination of the equivalent emission source, when the right technics are applied. These technics 
are described in [3]-[9].

The equivalent emission sources may be represented, as a first approach, by magnetic and electric dipoles that can be determined through the application of van Veen and Bergervöt Antenna, Kanda Antenna or Multipole Antenna.

The measurement of the magnetic dipole is normally carried out, since these antennas' measurements happen in the near field region, where the magnetic fields are much more representative than electric fields [7]. Especially in some cases, the measurement of electric fields cannot be precisely performed [10].

On the other hand, as can be verified on EMC Standards, as [1] and [2], the emission limits are represented by electric fields, in most of the cases. Thus, it is important to obtain the electric fields emitted by an arbitrary source, in order to check the whole electromagnetic scenario. To overcome this gap, a hybrid technic is proposed to calculate the electric fields from the magnetic fields, based on measurements and Analytical/FDTD calculation method [10].

\section{OBTAINING EQUIVALENT EMISSION SOURCES BY LLAS}

As already mentioned, some types of LLA are being studied for obtaining equivalent emission sources. The most known of them are: van Veen and Bergervöt Antenna, Kanda Antenna and Multipole Antenna. All of these antennas have some common characteristics, being composed by large loops, that surrounds the Device Under Test (DUT). The electric currents measured in these loops are induced by the electromagnetic fields emitted by the DUT. From these measured currents and with the right technics applied, the DUT's equivalent emission source is obtained.

The general process for obtaining the equivalent emission sources, is shown below, in Fig, 1.

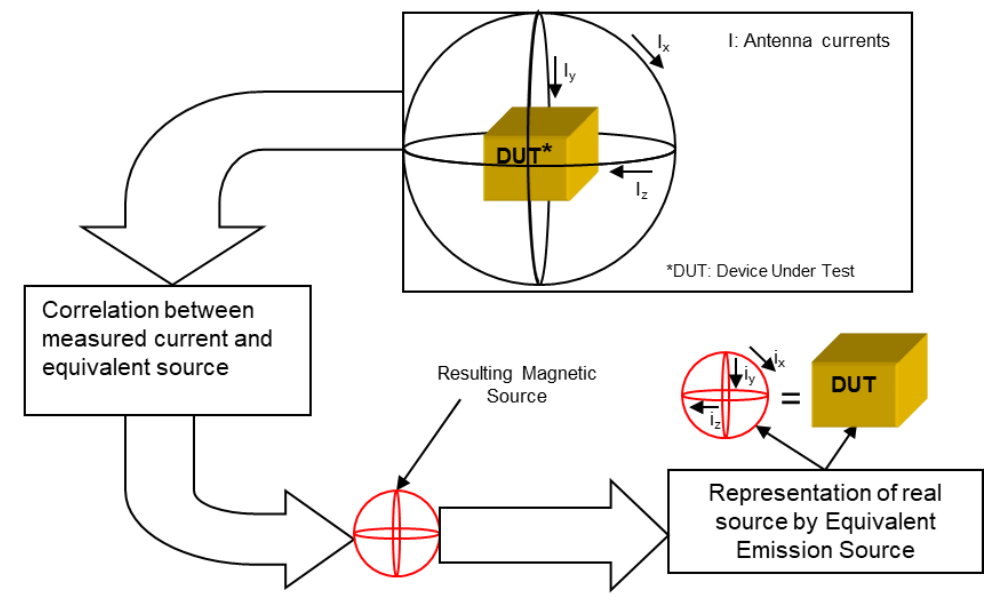

Fig. 1. DUT to Equivalent Emission Source transformation process by LLA measurement

From the picture above, the current of the magnetic dipole $\left(i_{n}\right)$ is calculated from the current measured in loop $\left(\mathrm{I}_{\mathrm{n}}\right)$, for $\mathrm{n}=\mathrm{x}, \mathrm{y}$ and $\mathrm{z}$. However, the total DUT representation shall be composed by the three magnetic dipoles supplied by currents $i_{x}, i_{y}$ and $i_{z}$.

It is briefly described how to obtain the equivalent emission source with the application of main LLAs: van Veen and Bergervöt Antenna, Kanda Antenna and Multipolar Antenna. 


\section{A. The van Veen and Bergervöt Antenna}

The van Veen and Bergervöt Antenna is represented in Fig. 2 [5].

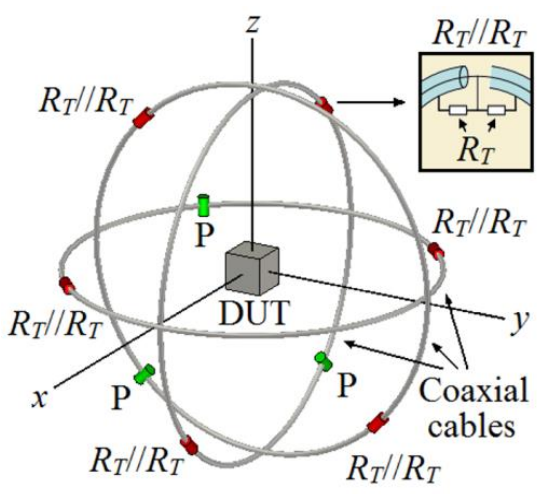

Fig. 2. The van Veen and Bergervöt Antenna Representation

This antenna is composed by three large loops, orthogonally disposed, assembled with coaxial cables. The current measurement is made on "P" points, shown in the picture above, directly with a current probe. The representation of the DUT measured by this antenna is only possible by magnetic dipoles, so it does not have capacity of electric dipoles representation and multipole "filtering" ( $3^{\text {rd }}$ order element - octupole - influences in dipole measurement) [7].

The DUT is placed inside this antenna, generating a current $\mathrm{I}_{\mathrm{Pn}}$ that is the current measured in the point "P" of each loop. Each loop is represented by the index $\mathrm{n}=\mathrm{x}, \mathrm{y}$ or $\mathrm{z}$.

The induced current $\mathrm{I}_{\mathrm{An}}$ is calculated by:

$$
I_{A_{n}}=I_{P_{n}}\left\{\begin{array}{l}
\frac{R_{C}}{R_{T}} \operatorname{sen}\left(\frac{k_{C} \pi b}{2}\right)+\mathrm{j} \cos \left(\frac{k_{C} \pi b}{2}\right)+ \\
+\mathrm{j} \frac{2 \pi R_{C}}{\mu \mathrm{c}}\left[\ln \left(\frac{8 b}{a_{C}}\right)-2\right] \frac{\operatorname{sen}\left(\frac{k_{C} \pi b}{2}\right)}{\tan \left(\frac{k \pi b}{2}\right)}
\end{array}\right\}
$$

Where $I_{P n}$ is the measured current, $R_{C}$ is the coaxial cable characteristic impedance, $R_{T}$ is the termination impedance, $\mathrm{k}_{\mathrm{c}}$ is the propagation constant, $\mu$ is the magnetic permeability, $\mathrm{a}_{\mathrm{c}}$ is the coaxial cable sectional radius and $\mathrm{b}$ is the large loop radius.

As detailed in [6], the magnetic dipole current $\left(i_{n}\right)$, is so obtained by:

$$
i_{n}=\frac{I_{A_{n}} 2 b L_{A}}{\mu_{0} \pi q^{2}}
$$

The current $i_{n}$, where $n=x, y$ or $z$, shall be applied to the three magnetic dipoles that composes the equivalent emission source, as shown in figure 1.

This current only refers to the magnetic component of the DUT's emission. The electric component is excluded, due to this antenna's characteristics [6]. 


\section{B. The Kanda Antenna}

The Kanda Antenna is represented in Fig. 3 [3], [4].

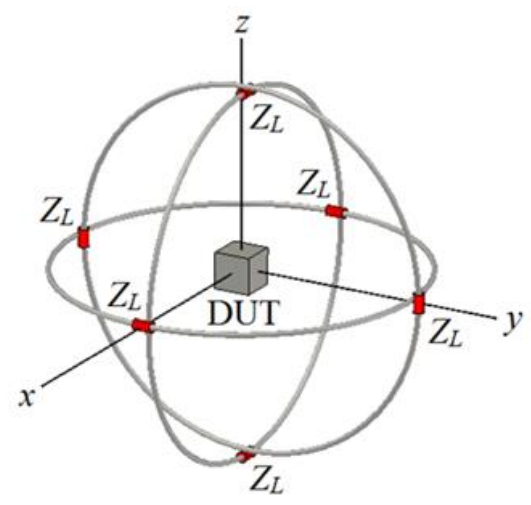

Fig. 3. Kanda Antenna Representation

This antenna is composed by three large loops, orthogonally disposed, assembled with rigid wires or curved metallic pipes. It requires external processing for total measured current in each loop, what adds a difficulty on its application. It has the capacity of DUT representation by magnetic dipole and/or electric dipole, but does not have capacity of multipole "filtering" ( $3{ }^{\text {rd }}$ order element - octupole - influences in dipole measurement).

To obtain the magnetic dipole, the DUT is placed inside this antenna, resulting on $\mathrm{I}_{\sum \mathrm{n}}$, that is the sum of the currents measured in the points " 0 " $\left(\mathrm{I}_{0 \mathrm{n}}\right)$ and " $\pi$ " $\left(\mathrm{I}_{\pi \mathrm{n}}\right)$ of each loop. Each loop is represented by the index $\mathrm{n}=\mathrm{x}$, y or $\mathrm{z}$.

$$
I_{\Sigma_{n}}=\frac{I_{0_{n}}+I_{\pi_{n}}}{2}=\frac{2 \pi b m_{M_{n}} G_{M} Y_{0}}{1+2 Y_{0} Z_{L}}
$$

With some mathematical arrangements [3], [4] the magnetic moment of the magnetic dipole can be obtained by:

$$
m_{M_{n}}=2 I_{\Sigma_{n}} \frac{\mathrm{j} \eta k b[\ln (8 b / a)-2]+2 Z_{L}}{\eta k^{2}[1+1 /(\mathrm{j} k b)] e^{-\mathrm{j} k b}}
$$

Finally the magnetic dipoles currents, for each one of the three loops, are obtained by:

$$
i_{n}=\frac{m_{M_{n}}}{\pi q^{2}}
$$

The current of each magnetic dipole here is obtained with regards only to the magnetic moment of the measurement. Similarly, an additional part, related to the electric moment, can be obtained by $\mathrm{I}_{\Delta \mathrm{n}}$ calculation [3], [4].

In order to keep similarity with the DUT's representation by van Veen and Bergervöt Antenna, which can only obtain the magnetic part, the electric part is disregarded.

\section{The Multipolar Antenna}

The Multipolar Antenna is represented in Fig. 4 [7], [8]. 


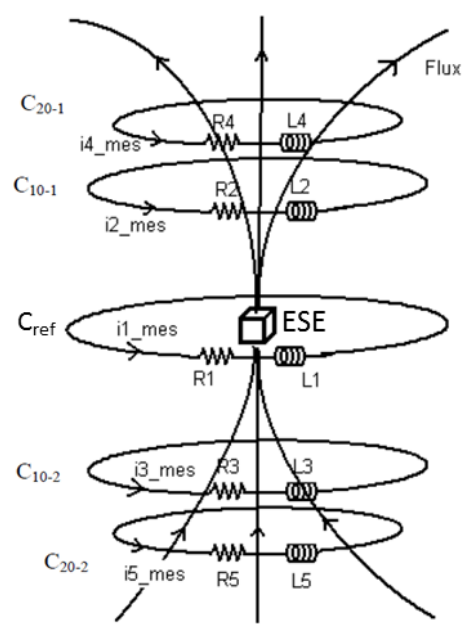

Source: S. Zangui [8]

Fig. 4. Multipolar Antenna Representation

This antenna is composed by large loops, concentrically disposed, forming a sphere. It is assembled with rigid wires. The current is measured directly on the loop without necessity of external processing. The DUT is represented by magnetic multipoles. It has the capacity of multipole "filtering" (separate measurement for $1^{\text {st }}$ and $2^{\text {nd }}$ orders elements).

For the magnetic dipole obtaining, the DUT is measured in this antenna, resulting on coefficient $\mathrm{A}_{10}$, as follows:

$$
A_{10}=\frac{10^{8} r_{M}}{32 \pi}\left(\phi_{10 \_1}-\phi_{10 \_}\right)=2,24.10^{5}\left(\phi_{10 \_1}-\phi_{10 \_}\right)
$$

Where $r_{M}$ is the antenna's sphere radius and $\phi_{10 \_n}$ is the magnetic flux of the $C_{10 \_n}$ loop.

The flux is calculated from the measured current (I), by:

$$
\phi_{10 \_n}=I_{10 \_n} . L
$$

Where $\mathrm{L}$ is the loop inductance, that is obtained by:

$$
L=\mu_{0} b\left(\ln \left(\frac{8 b}{r_{c}}\right)-\frac{7}{4}\right)
$$

Finally, to obtain the current for each loop that represents the DUT:

$$
i_{n}=\frac{\pi r^{2}}{A_{10}}
$$

\section{Electromagnetic Fields Analytical Calculation}

With the currents measured by any of the antennas mentioned above, the magnetic dipole can be determined (with calculated currents $i_{x}, i_{y}$ and $i_{z}$, for each loop as in fig. 1). Single loop is represented in Fig. 5. 


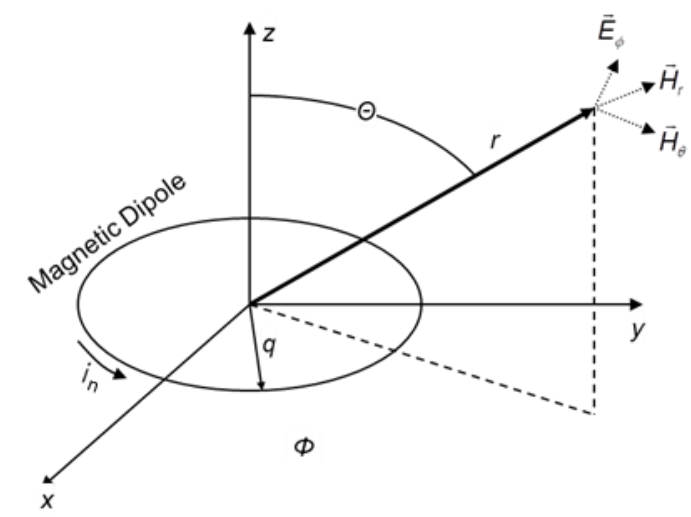

Fig. 5. Electromagnetic Fields Emitted by a Magnetic Dipole

And the fields can be calculated by, for each single loop (in spherical coordinates) [11]:

$$
\begin{gathered}
H_{r}=j \frac{k q^{2} i \cos \theta}{2 r^{2}}\left(1+\frac{1}{j k r}\right) e^{-j k r} \\
H_{\theta}=-\frac{(k q)^{2} i \operatorname{sen} \theta}{4 r}\left(1+\frac{1}{j k r}-\frac{1}{(k r)^{2}}\right) e^{-j k r} \\
H_{\phi}=0 \\
E_{r}=E_{\theta}=0 \\
E_{\phi}=\eta j \frac{(k q)^{2} i \operatorname{sen} \theta}{4 r}\left(1+\frac{1}{j k r}\right) e^{-j k r}
\end{gathered}
$$

Converting to rectangular coordinates:

$$
\begin{aligned}
& H_{x}=\operatorname{sen} \theta \cos \phi H_{r}+\cos \theta \cos \phi H_{\theta}-\operatorname{sen} \phi H_{\phi} \\
& H_{y}=\operatorname{sen} \theta \operatorname{sen} \phi H_{r}+\cos \theta \operatorname{sen} \phi H_{\theta}+\cos \phi H_{\phi} \\
& H_{z}=\cos \theta H_{r}-\operatorname{sen} \theta H_{\theta} \\
& E_{x}=\operatorname{sen} \theta \cos \phi E_{r}+\cos \theta \cos \phi E_{\theta}-\operatorname{sen} \phi E_{\phi} \\
& E_{y}=\operatorname{sen} \theta \operatorname{sen} \phi E_{r}+\cos \theta \operatorname{sen} \phi E_{\theta}+\cos \phi E_{\phi} \\
& E_{z}=\cos \theta E_{r}-\operatorname{sen} \theta E_{\theta}
\end{aligned}
$$

\section{E. Analytical/FDTD Hybrid Method for Electric Field calculation}

As an alternative to the aforementioned analytical calculation, this hybrid method is presented.

From [10] this method has been proposed, specifically for electric fields calculation, from atmospheric discharges.

As a great contribution, this method allows the calculation of the electric fields (E) from the magnetic flow density vector (B) that can be obtained by measurements and analytical or numerical calculations.

A simplified schematic represents how this calculation shall be prepared is shown in Fig. 6 . 

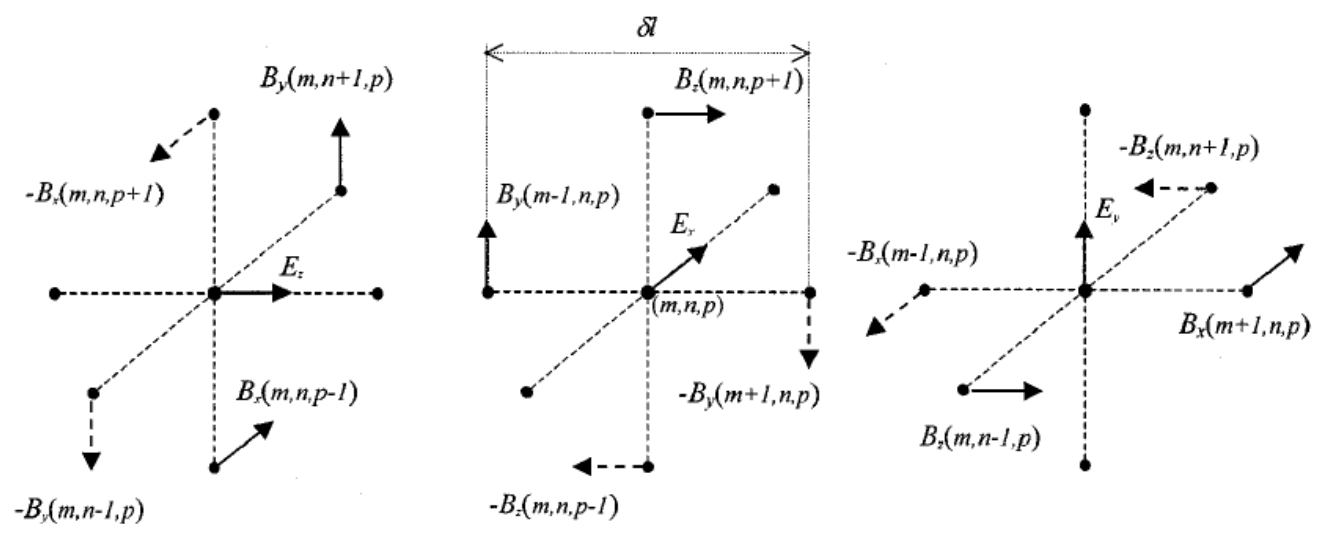

Source: C. Sartori and J. Cardoso [10]

Fig. 6. Representation of electrical fields obtaining from magnetic flow density vectors

The magnetic flow density vector (B) in each point of space shall be calculated from the magnetic field $(\mathrm{H})$ emitted by a magnetic dipole, which can be calculated analytically (equations (10), (11), (12) and (15)). B is obtained by:

$$
\vec{B}_{n}=H_{n} \mu
$$

For one point of space, $\mathrm{E}_{\mathrm{x}}, \mathrm{E}_{\mathrm{y}}$ and $\mathrm{E}_{\mathrm{z}}$, are calculated from components $\mathrm{B}$ around to this point.

Also, the distance between these points $(\delta 1)$ is important for this method, calculated by :

$$
\delta l \leq 0,01 R
$$

Where, R is the distance from the DUT's center to the chosen measurement point on space.

$$
\begin{aligned}
& k+\frac{1}{2} E_{z}(m, n, p) \\
& =k-\frac{1}{2} E_{z}(m, n, p)+\frac{c^{2} \Delta t}{\delta l}\left[k B_{y}(m, n+1, p)\right. \\
& \left.-k B_{y}(m, n-1, p)+k B_{x}(m, n, p-1)-k B_{y}(m, n, p+1)\right] \\
& k+\frac{1}{2} E_{x}(m, n, p) \\
& =k-\frac{1}{2} E_{x}(m, n, p)+\frac{c^{2} \Delta t}{\delta l}\left[k B_{z}(m, n, p+1)\right. \\
& \left.-k B_{z}(m, n, p-1)+k B_{y}(m-1, n, p)-k B_{y}(m+1, n, p)\right]
\end{aligned}
$$


The time-step factor "k" shall be defined by:

$$
\Delta t \leq \frac{\delta l}{2 c}
$$

With these parameters, a mathematical tool may be used to calculate it, with time-steps processing.

Finally, the components of electric fields are calculated $\left(E_{x}, E_{y}\right.$ and $\left.E_{z}\right)$. A representation of $E_{z}$ compared to $\mathrm{B}_{\mathrm{y}}$, is shown in Fig. 7.

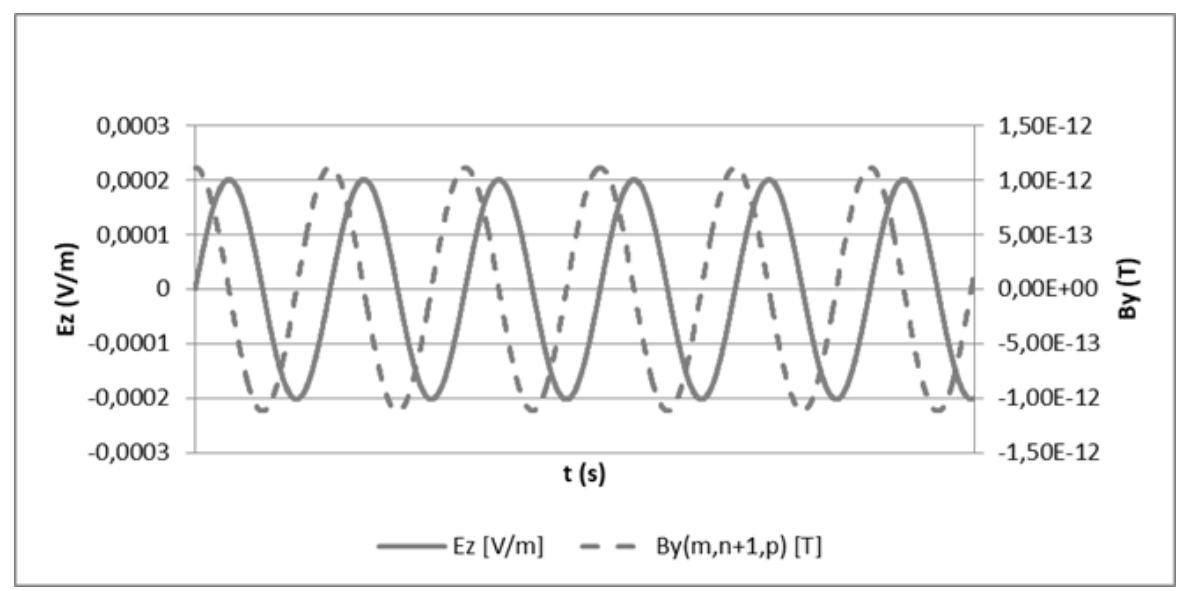

Fig. 7. Example of electric field $\left(\mathrm{E}_{\mathrm{z}}\right)$ resulting from calculation and comparison with magnetic flow density vector $\left(\mathrm{B}_{\mathrm{y}}\right)$.

\section{EXPERIMENTAL VALIDATION}

In this chapter a comparison between three methods is shown: analytical method, numerical, method and hybrid method, this last one is the focus of this paper.

The general process of experimental validation is summarized in Fig. 8, for a simpler understanding of the process.

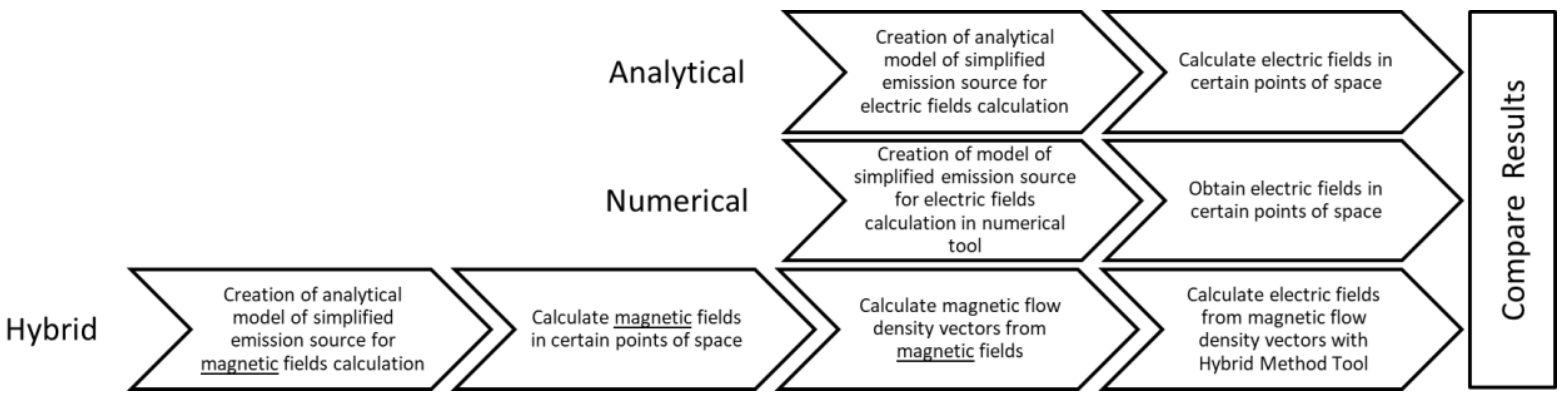

Fig. 8. Visual chart of experimental validation process

The first step is the equivalent magnetic dipole current obtaining, that shall be made with one of the antennas presented in chapter II. Due to the availability and most simplified construction, the Kanda Antenna has been utilized for the experimental validation. Originally the Kanda Antenna is composed by 3-loop, orthogonally disposed, as shown before. But only one loop is considered, and, conceptually, it is enough for this validation. For a complete Kanda Antenna utilization, this whole procedure should be executed three times, once for each loop, as shown in Fig. 9.

From the measurements of a generic device under test (DUT) with Kanda Antenna, the values for Brazilian Microwave and Optoelectronics Society-SBMO received 18 Sep 2017; for review 18 Sep 2017; accepted 14 Dec 2017 7 Brazilian Society of Electromagnetism-SBMag 
induced current from magnetic dipole $\left(\mathrm{I}_{\Sigma}\right)$ were calculated.

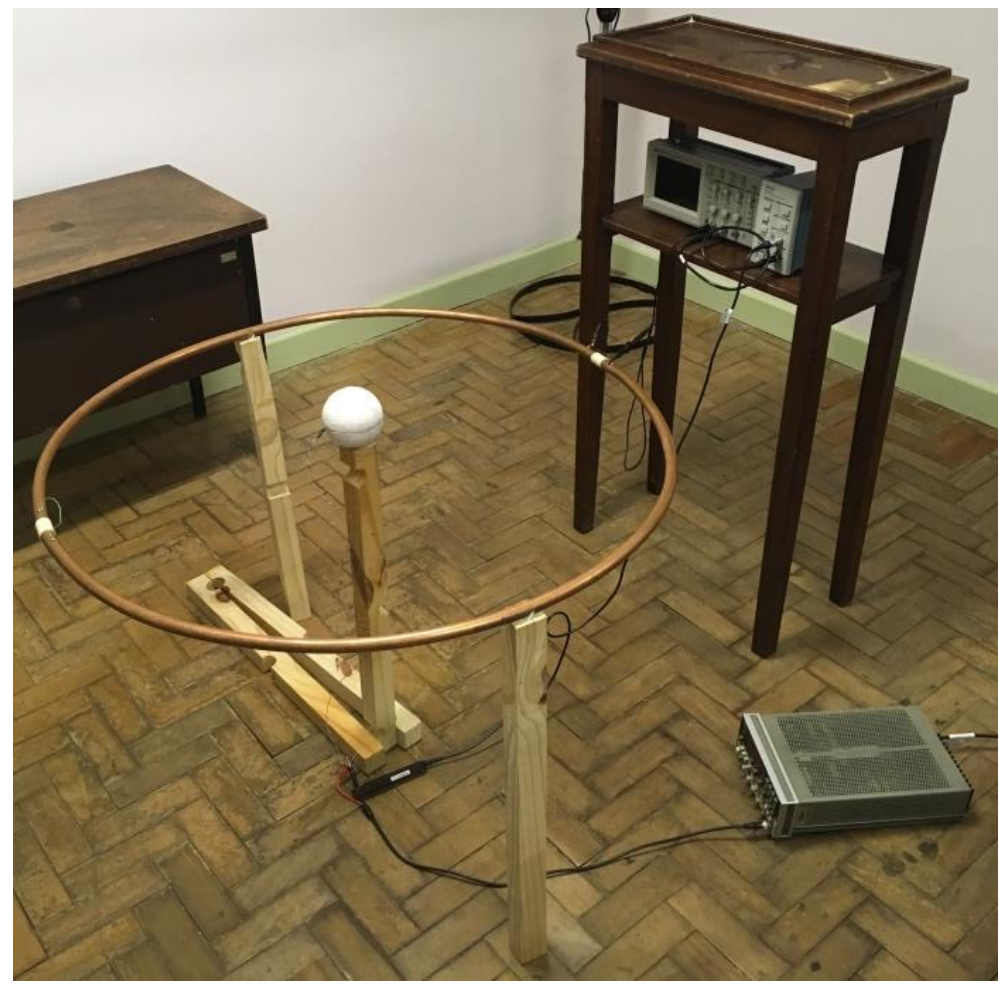

Fig. 9. Test setup with Kanda Antenna and a small current loop (as the DUT)

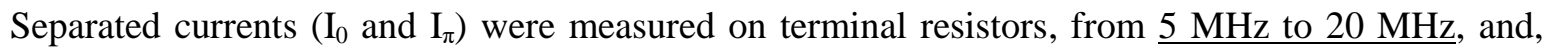
after external processing, the final induced current $\mathrm{I}_{\Sigma}$ resulted on (as shown in chapter II-B, equation (3)) the curve shown in Fig. 10.

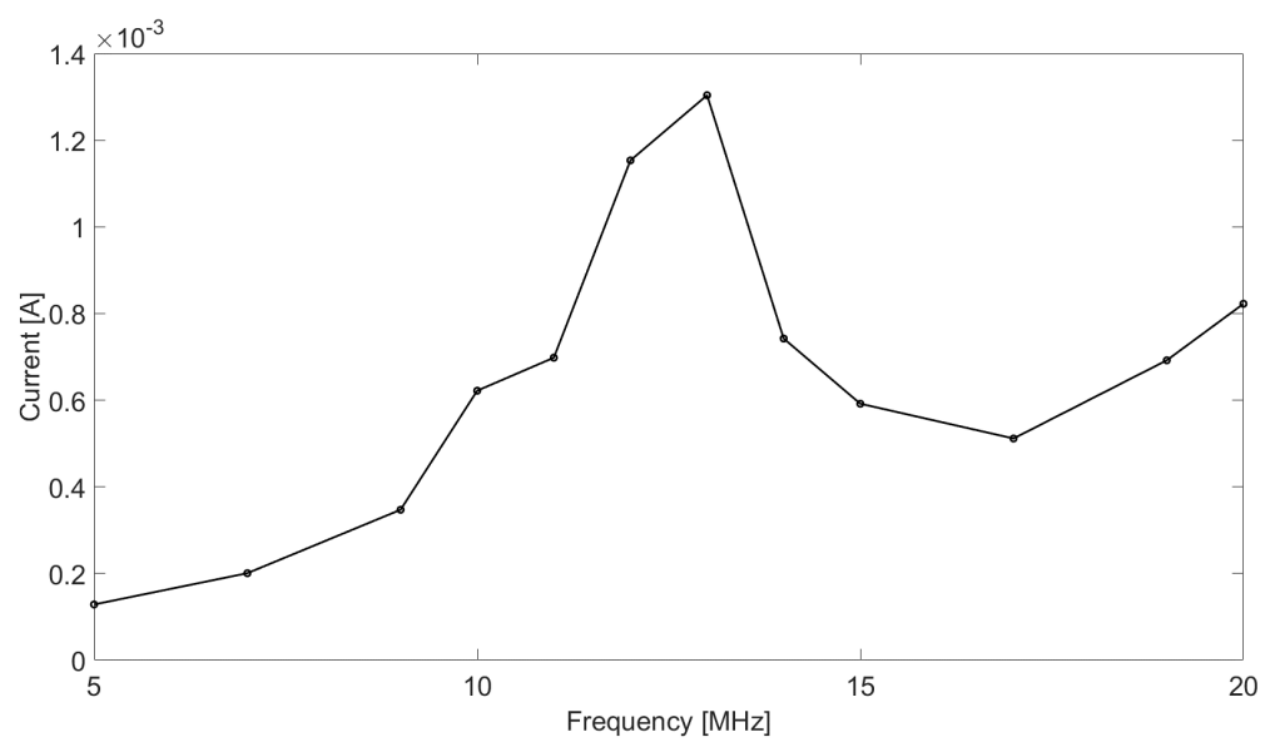

Fig. 10. Kanda Antenna Current $\left(\mathrm{I}_{\Sigma}\right)$ measured and processed

From the equations (3), (4) and (5), the equivalent magnetic dipole current ( $\left.i_{x}\right)$ was obtained and shown in Fig. 11. 


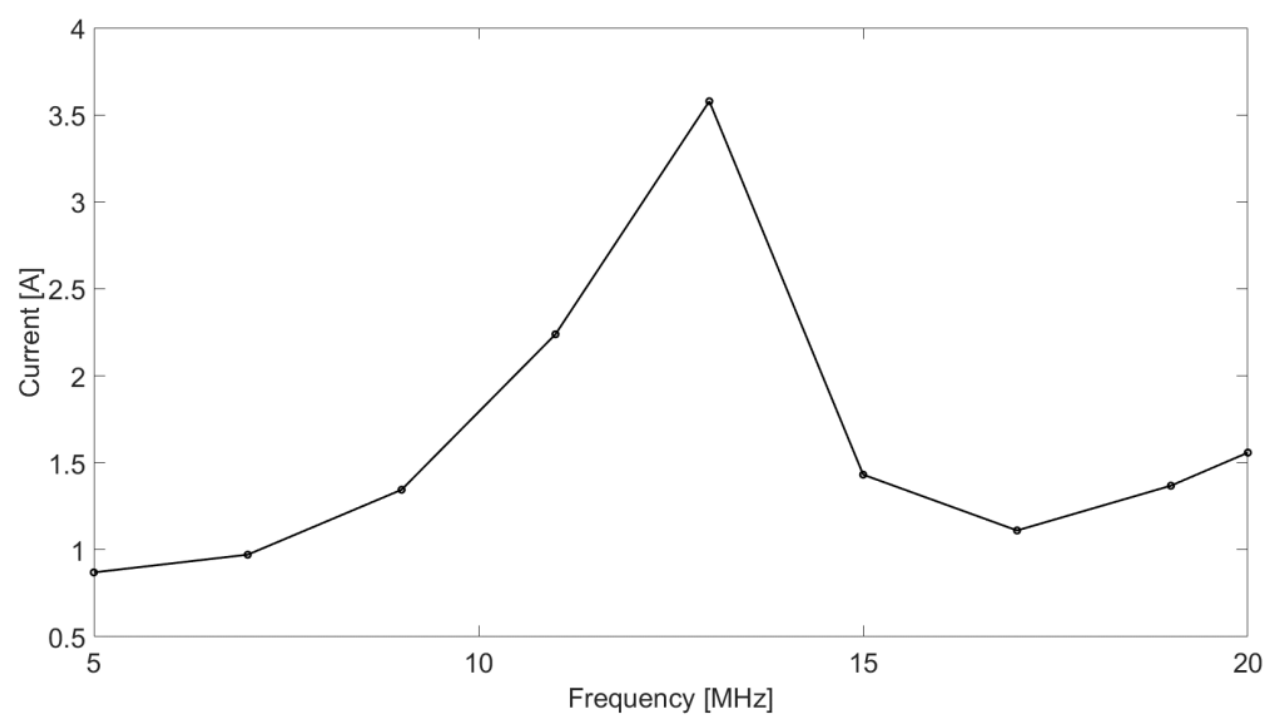

Fig. 11. Equivalent magnetic dipole current $\left(i_{x}\right)$ calculated

For an application with a complete Kanda Antenna, this process should be repeated, to obtain the currents $i_{y}$ and $i_{z}$, for the antenna loops " $y$ " and "z", and respective equivalent magnetic loop " $y$ " and “z” (see fig. 1).

From the equivalent magnetic dipole current shown above, the magnetic and electric fields emitted by the magnetic dipole were analytically calculated in many points of space, from equations (10)-(16).

As already mentioned, the electric fields are alternatively calculated by a Hybrid Method. For that, the magnetic flow density vector (B) was calculated from the magnetic fields $(\mathrm{H})$ and from these values it was calculated the electric fields (E).

Additionally, to enrich the comparison, two numerical models were created in a numerical simulation tool [12]. These models have been intensively evaluated and several iterations have been ran, in order to have the most appropriated models for this comparison, resulting in two models, one composed by 11.000 tetrahedrons (model "a"), more simple (but faster, for computer processing) and another one composed by 500.000 tetrahedrons (model "b"), more detailed (but much slower, for computer processing).

Figure 12 represents both models (only the quantity of elements is different).

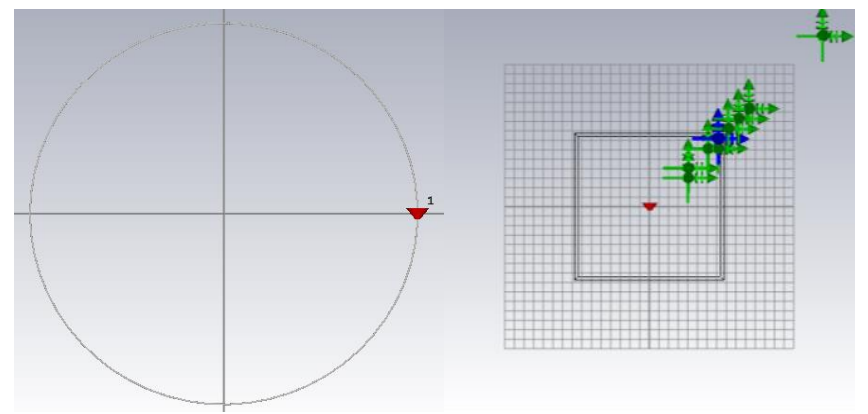

Fig. 12. Numerical models. Magnetic Dipole (1) and Electric Fields Probes (2)

Finally, a comparison between the values of electric fields calculated by Analytical, Hybrid and 
Numerical ("a" and "b" models) is shown in Fig. 13, with the results in $5 \mathrm{MHz}$ and $20 \mathrm{MHz}$, calculated in some points of space ranging from 5.8 meters to 29.4 meters (distances from the center of the magnetic dipole to the measurement points).

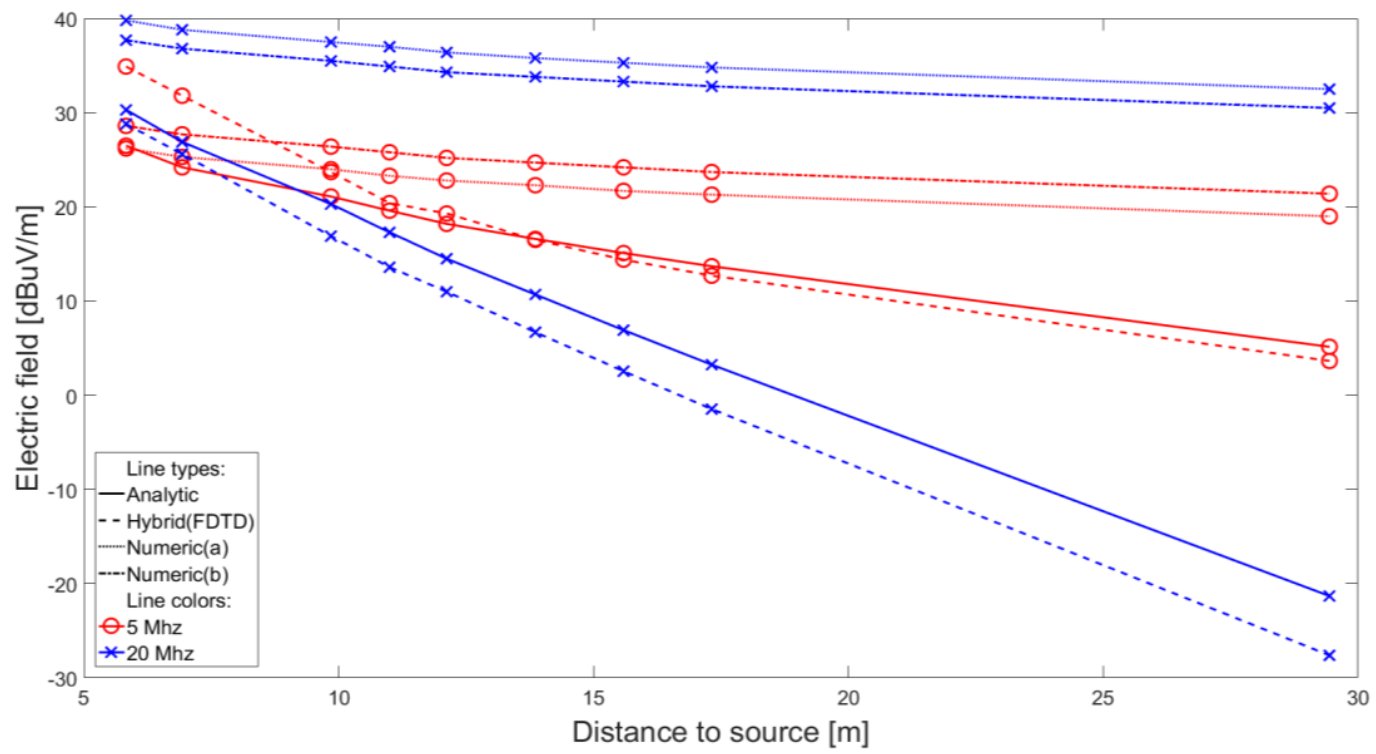

Fig. 13. Total electric field (5 MHz and $20 \mathrm{MHz}$ ) calculated by analytical, hybrid and numerical methods

The same was calculated for others frequencies, ranging from $5 \mathrm{MHz}$ to $20 \mathrm{MHz}$. The 3D charts show the whole range, in Fig. 14, with analytical method, hybrid method, numerical model "a" and numerical model "b".
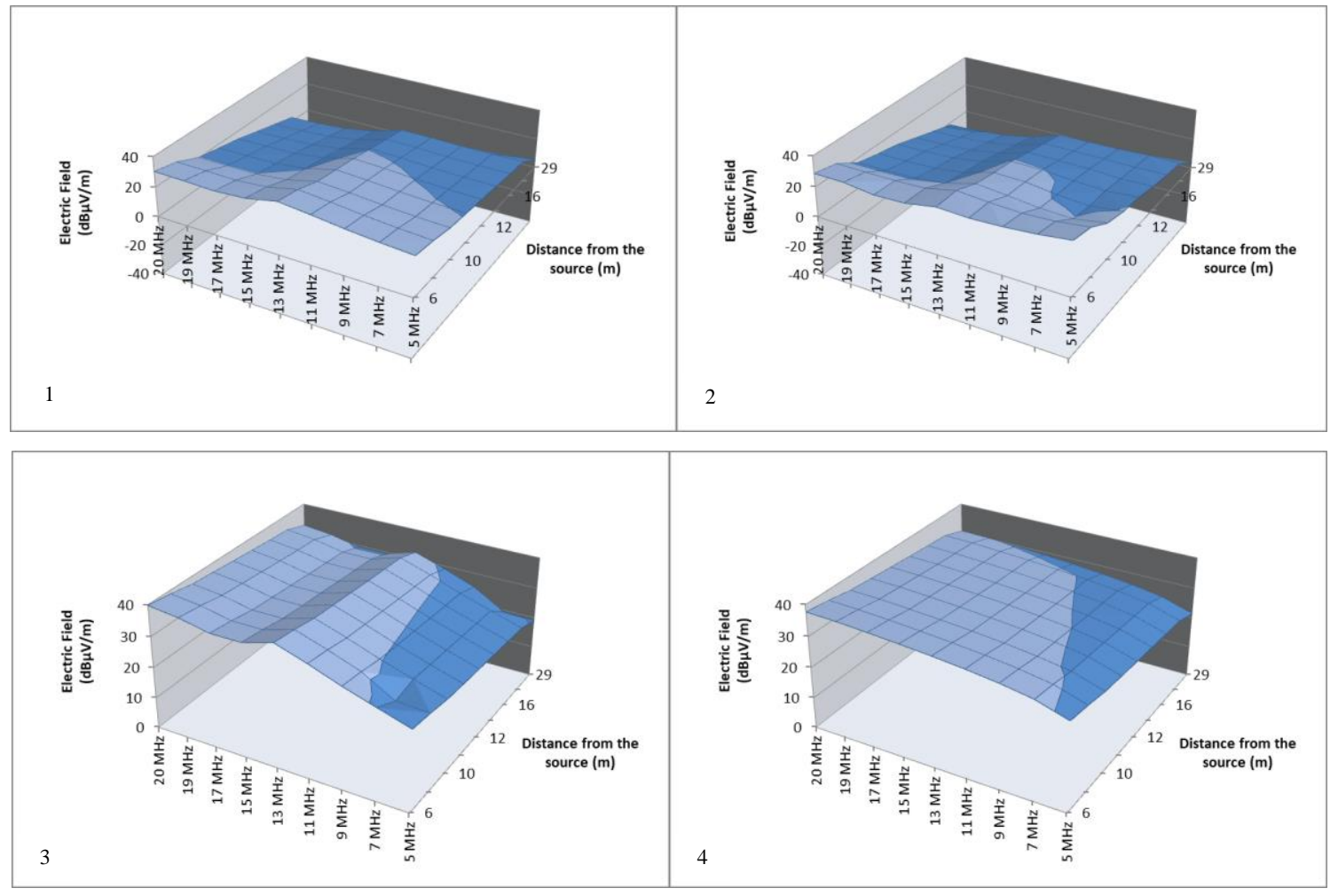

Fig. 14. 3D charts of total electric field from $5 \mathrm{MHz}$ to $20 \mathrm{MHz}$ calculated by analytical (1), hybrid (2) and numerical methods ("a" (3) and "b" (4) numerical models) 


\section{Discussion On Method Results}

In the last chapter three different methods have been compared, analytical method, numerical method and hybrid method, and from the results shown above, it can be emphasized that the proposed hybrid method, when compared to analytical method, shows a good relation and precision (difference between 0.05 to $6 \mathrm{~dB} \mu \mathrm{V} / \mathrm{m}$ with $0.85 \mathrm{~dB} \mu \mathrm{V} / \mathrm{m}$ in average). The differences in $20 \mathrm{MHz}$ are bigger than in $5 \mathrm{MHz}$, due to the dimensional characteristics of the hybrid method. By the other side, the numerical method showed a bigger difference when compared to analytical calculation (difference about $15 \mathrm{~dB} \mu \mathrm{V} / \mathrm{m}$, in average). This result is related to the numerical modeling characteristics, as the model shape, solver type, etc., and to the computer capacity.

However, it can be concluded that the proposed hybrid method is applicable for the electric fields calculation.

\section{CONCLUSIONS}

Initially, it can be mentioned the relevance of DUT's representation by equivalent emission sources, as represented in this paper, considering its ease construction, lacking any complex geometry and thus very easily represented in a simulation model.

Any of the LLAs here presented are able to be applied with this method, each one with some different characteristics, but all of them are applicable. Multipolar Antenna represents the last status of development for equivalent emission sources determination, since it separates the multipole characteristics of a DUT, increasing the precision when rebuilding it by magnetic dipoles, quadrupoles, octupoles, etc.

Regarding to the electric fields, the results obtained by the proposed hybrid method can offer a better approximation than the ones obtained by numerical simulation modeling. Considering that the obtaining of electric fields with a good precision is a real necessity, the application of the proposed hybrid method shows an advance in this field of study. This method is especially useful because the input for this method is the magnetic flow density vector, which can be obtained by measurement or calculation, regardless of the emission source type.

However, the application of the hybrid method, together with measurements by LLAs, represents a good and applicable method for the representation of DUTs by equivalent emission sources, in order to allow the assessment of electromagnetic environments that can be especially suitable for electromagnetic compatibility evaluations.

\section{REFERENCES}

[1] CISPR 25: 2008-03, Vehicles, boats and internal combustion engines - Radio disturbance characteristics - Limits and methods of measurement for the protection of on-board receivers.

[2] ISO 11452: 2005-02, Road vehicles - Component test methods for electrical disturbances from narrowband radiated electromagnetic energy.

[3] M. Kanda and D. Hill, "A three-loop method for determining the radiation characteristics of an electrically small source", IEEE Transactions on Electromagnetic Compatibility, vol. 34, no. 1, Aug. 1992.

[4] M. Kanda et al, "An Electromagnetic Near-Field Sensor for Simultaneous Electric and Magnetic-Field Measurements", IEEE Transactions on Electromagnetic Compatibility, vol. 26, no. 3, pp. 102-110, Aug. 1984. 
[5] J. R. Bergervöt and H. v. Veen, “A Large-Loop Antenna for Magnetic Field Measurements", Zurich International Symposium on EMC, Proceedings, 1989.

[6] D. Cardia, “Aplicação Da Antena de Van Veen E Bergervöt Na Análise De Contribuições De Fontes De Emissão Radiada Em Ambientes Eletromagnéticos”, Dissertação de Mestrado, Universidade de São Paulo, 2010.

[7] B. Vincent, "Identification de sources eletromagnétiques multipolaires équivalentes par filtrage spatial: Application à la CEM rayonée pour le convertisseurs d'eletronique de puissance", These, L'Institut polytechnique de Grenoble, 2009.

[8] S. Zangui, "Determination et Modelisation du Couplage en Champ Proche Magnetique entre Systemes Complexes", These, Université de Lyon, 2011.

[9] A. Bréard et al, "Overview on the Evolution of Near Magnetic Field Coupling Prediction Using Equivalent Multipole Spherical Harmonic Sources", IEEE Transactions on Electromagnetic Compatibility, vol. 59, no. 2, pp. 584-592, 2017.

[10] C. Sartori and J. Cardoso, "An Analytical-FDTD Method for Near LEMP Calculation", IEEE Transactions on Magnetics, vol. 36, no. 4, pp. 1631-1634, 2000.

[11] C. Balanis, "Antenna Theory: Analysis and Design", $2^{a}$ Edição, Wiley.

[12] CST Microwave Studio, Software, Version 2016. 